\title{
Is exercise an efficacious treatment for depression? A comment upon recent negative findings
}

\section{Felipe Barreto Schuch* and Marcelo Pio de Almeida Fleck}

Post-Graduate Program in Medical Science: Psychiatry, Hospital de Clínicas de Porto Alegre, Universidade Federal do Rio Grande do Sul, Porto Alegre, Brazil *Correspondence: felipe.schuch@ufrgs.br

Edited by:

Eduardo Lusa Cadore, Federal University of Rio Grande do Sul, Brazil

Exercise is receiving substantial and increasing attention as a potential treatment for depression. Despite the many positive meta-analytical findings and recommendations of some guidelines to incorporate exercise as a treatment for depression (National Institute for Health and Clinical Excellence, 2009), most clinical trials have significant methodological flaws that limit the generalizability of their findings (Daley, 2008; Rethorst et al., 2009). Furthermore, recent meta-analyses show that, when only robust clinical trials are included, the effects of exercise are "moderate at best" or statistically insignificant (Rimer et al., 2012).

Similarly, four recent, robust, randomized controlled trials (RCTs) have failed to find any antidepressant effects of exercise: the DEMO (Krogh et al., 2009), DEMO II (Krogh et al., 2012), TReatment with Exercise Augmentation for Depression (TREAD) (Trivedi et al., 2011), and TREAD-UK (Chalder et al., 2012). The DEMO trial (Krogh et al., 2009) compared aerobic exercise, anaerobic exercise, and relaxation (control) groups; after 4 months of intervention, no differences in depressive symptoms, as assessed by the Hamilton scale for depression (HAM-D17) (Hamilton, 1967), were found between the groups. Subsequently, the DEMO II trial (Krogh et al., 2012) compared aerobic exercise and stretching (control) groups; after 3 months, the authors found reductions in HAM-D17 scores in both groups but failed to find differences between the groups. In both studies, the authors concluded that exercise was as effective as placebo and had no biological antidepressant effects.

Corroborating the DEMO and DEMO II findings, the TREAD (Trivedi et al., 2011) found a non-significant trend $(p<0.06)$ toward a remission rate of $16 \mathrm{kcal} / \mathrm{kg} /$ week for aerobic exercise versus $4 \mathrm{kcal} /$ $\mathrm{kg} /$ week for aerobic exercise (control) after 3 months of intervention. Lastly, the TREAting Depression with physical activity (TREAD-UK) (Chalder et al., 2012) evaluated the cost-effectiveness of a strategy for promoting and increasing the physical activity levels of depressed patients in primary care. After 8 months, physical activity was shown to be an ineffective and more costly strategy than conventional primary care assistance and resulted in a non-costeffective strategy according to willingnessto-pay thresholds.

Considering these recent results, the answer to the question "is exercise an efficacious treatment for depression?" appears to be "No." However, before this question is answered, some issues must be highlighted.

Similar to the findings regarding exercise, several meta-analyses have shown that the benefits of antidepressants are "minimal or non-existent," as they are as effective as placebo. Other meta-analyses have shown that antidepressants are effective only in severe, but not in moderate or mild depression (Kirsch et al., 2008; Fournier et al., 2010; Khan et al., 2012). On the other hand, clinical practice and some qualitative studies have revealed that both exercise and antidepressants are effective in the opinion of patients (Searle et al., 2011) and mental health professionals (Martinsen, 1994; Kirsch, 2008); these findings reveal a discrepancy between the views of health professionals and patients and the clinical findings.

There are some possible explanations for this discrepancy: (1) the heterogeneity of "depression" as a construct; (2) the psychometric pitfalls of depression assessment scales; and (3) the possible interference of unspecific factors that could mask the biological effects of exercise.

Parker (2005) argued that the "major depression" concept classifies a heterogeneous group of patients who may have different, and sometimes opposite, clinical features and symptoms (e.g., insomnia or hypersomnia, weight loss or weight gain, and psychomotor retardation or psychomotor agitation) into on diagnosis. In the words of Parker, the major depression construct "circumscribes a range of heterogeneous conditions, homogenizes them, and has come to be viewed as an entity." This quote exemplifies the diagnoses of individuals with "clinical dyspnea" because such diagnoses are not informative because the individual may have asthma, pneumonia, or a pulmonary embolus, and each condition requires a different treatment. Similarly, major depression may need a more finely focused diagnosis that requires greater explanatory power. The proposal of Parker is a new categorical-dimensional model that classifies three more finely focused diagnostic subgroups: psychotic depression, melancholic depression, and non-melancholic depression; these subgroups possibly have different biological backgrounds and may be responsive to different treatments. For more detailed information, see Parker's works concerning a new categorical-dimensional construct of depression.

Another possible explanation for this discrepancy may be the low "effectivity" of the HAM-D17, which is the most used instrument in RCTs and was used in three of the recent studies of exercise (DEMO, DEMO II, and TREAD). Some studies have shown that this scale has several flaws, as assessed by classical (Bagby et al., 2004; Fleck et al., 2004) and modern psychometric techniques, including Item response theory and Rasch analyses (Bagby et al., 2004). Based upon these studies, the HAM-D17 lacks unidimensionality; furthermore, some items appear to be insufficiently discriminative of different levels of depression, particularly within the mild and moderate levels, although the HAM-D17s work for severe 
levels of depression (Santor and Coyne, 2001). According Salum et al. (2011), the HAM-D17 is like an "industrial thermometer being used to measure a temperature of a baby." Interestingly, antidepressants have higher efficacy in severe, compared to low or moderate, depression (Kirsch et al., 2008; Fournier et al., 2010). Furthermore, some preliminary results show that exercise is also effective as a complementary treatment of severely depressed inpatients (Knubben et al., 2007; Schuch et al., 2011).

Finally, exercise has many factors that "compose" the exercise session but are not exercise per se. For example, when a patient exercises in a group setting, or even in the company of health professionals, the resultant social support and attention may have an important role, especially in light depression. A similar effect occurs in structured psychotherapies studies, in which nonstructured interventions (placebos) efficacies similar to structured interventions (Jakobsen et al., 2011).

Before a final judgment is made, clinical trials using exercise as a treatment for depression must be carefully analyzed. Furthermore, meta-analyses show that exercise has similar efficacy as other standardized treatments for depression including some antidepressants and some psychotherapies. Thus, exercise may not be more efficacious than conventional treatments; however, it is not less efficacious.

On the other hand, exercise has other issues that deserve more attention: the initial acceptance and compliance/adherence. Two of the major difficulties of the use of exercise as a treatment for depressed patients are the initial acceptance of and compliance/adherence to exercise regimens. For example, two weaknesses of the DEMO II trial are the limited number of patients included and the low compliance with the exercise sessions.

In the DEMO trial, of 390 possible patients recruited, 100 refused to participate. In the DEMO II trial, the patients' inclusion had to stop due to "lower referral than anticipated." Second, the authors' initial sample size tests showed that 85 subjects in each group were necessary, but after the recruitment phase and an additional 12 months of recruitment, they had just 56 subjects in the aerobic exercise group and 59 in the stretching group. Lastly, in our study (unpublished data), 46 of 96 severely depressed inpatients refused to participate in the study, and, of these 46, 40 stated they had low interest in exercise.

Moreover, the in DEMO II trial, patients attended a mean 13.5 and 12.5 of 36 sessions of aerobic exercise and stretching, respectively. In an attempt to increase adherence/ compliance, Trivedi et al. (2006b) suggested that a more flexible program based upon energetic expenditure, without a fixed intensity (e.g., 70\% maximum heart rate), may be more suitable and increase compliance. For example, in the DOSE study (Trivedi et al., 2006a), patients in the exercise groups completed $72 \%$ of the sessions. In another recent study, Callaghan et al. (2011) showed that women that exercised "as recommended by national guidelines" attended 6 of 12 sessions (50\%), while the group that had exercised at the "preferred intensity" attended 8 of 12 sessions (66\%), resulting in a mean increase of $2 / 12$ sessions. In the TREAD (Trivedi et al., 2011) study, the low and high dose groups had mean adherences of 99.4 and $63.8 \%$, respectively. These three studies show that more flexible strategies may be an interesting alternative that may increase adherence and compliance compared to conventional strategies (e.g., $30 \mathrm{~min}$ at $70 \%$ of maximal heart rate).

Two interesting papers propose some practical suggestions to initiate and sustain exercise and physical activity in depressed patients. One is a paper from Seime and Vickers (2006) that suggests some practical strategies including the following: promote discussions related to what activities "would benefit your patient most based upon his/her symptoms" in terms of pre-preferences and personal barriers that prevent the patient from beginning or maintaining exercise, and share with patients an easily accessible brief overview of recommendations for physical activity that could include the guideline recommendations for public health and the dose-response relationship demonstrated by Dunn et al. (2005). Moreover, Blumenthal et al. (2012) suggested that exercise professionals should become more familiar with the principles of motivational interviewing, a well-researched approach to promoting behavioral change, and the "seven tips" for patients.

In summary, recent negative findings concerning the use of exercise as a treatment for depressed patients must be interpreted with caution. Exercise, along with other treatments for depression, has some issues because the instrument used to assess depression and the diagnoses of depression may lead to misunderstandings. Thus, the DSM-V will most likely open new perspectives concerning diagnoses of depression, and subsequently, the instruments used to assess depression. Although the initial adherence and compliance with exercise programs are a great challenge, the use of cognitive and motivational approaches and more flexible and comprehensive strategies of exercise may be useful in increasing and maintaining a the exercise of depressed patients.

\section{REFERENCES}

Bagby, R. M., Ryder, A. G., Schuller, D. R., and Marshall, M. B. (2004). The Hamilton Depression Rating Scale: has the gold standard become a lead weight? Am. J. Psychiatry 161, 2163-2177.

Blumenthal, J.A., Smith, P. J., and Hoffman, B. M. (2012). Opinion and evidence: is exercise a viable treatment for depression? ACSMs Health Fit. J. 16, 14-21.

Callaghan, P., Khalil, E., Morres, I., and Carter, T. (2011). Pragmatic randomised controlled trial of preferred intensity exercise in women living with depression. BMC Public Health 11:465. doi: 10.1186/1471-2458-11-465

Chalder, M., Wiles, N. J., Campbell, J., Hollinghurst, S. P., Searle, A., Haase, A. M., et al. (2012). A pragmatic randomised controlled trial to evaluate the cost-effectiveness of a physical activity intervention as a treatment for depression: the treating depression with physical activity (TREAD) trial. Health Technol. Assess. 16, 1-164.

Daley, A. (2008). Exercise and depression: a review of reviews. J. Clin. Psychol. Med. Settings 15, 140-147.

Dunn, A. L., Trivedi, M. H., Kampert, J. B., Clark, C. G., and Chambliss, H. O. (2005). Exercise treatment for depression: efficacy and dose response. Am. J. Prev. Med. 28, 1-8.

Fleck, M. P., Chaves, M. L., Poirier-Littre, M. F., Bourdel, M. C., Loo, H., and Guelfi, J. D. (2004). Depression in France and Brazil: factorial structure of the 17-item Hamilton Depression Scale in inpatients. J. Nerv. Ment. Dis. 192, 103-110.

Fournier, J. C., Derubeis, R. J., Hollon, S. D., Dimidjian, S., Amsterdam, J. D., Shelton, R. C., et al. (2010). Antidepressant drug effects and depression severity: a patient-level meta-analysis. JAMA 303, 47-53.

Hamilton, M. (1967). Development of a rating scale for primary depressive illness. Br. J. Soc. Clin. Psychol. 6, 278-296.

Jakobsen, J.C., Hansen, J.L., Storebo, O. J., Simonsen, E., and Gluud, C. (2011). The effects of cognitive therapy versus 'no intervention' for major depressive disorder. PLoS ONE 6:e28299. doi: 10.1371/journal.pone.0028299

Khan, A., Faucett, J., Lichtenberg, P., Kirsch, I., and Brown, W.A. (2012).A systematic review of comparative efficacy of treatments and controls for depression. PLoS ONE 7:e41778. doi: 10.1371/journal.pone.0041778

Kirsch, I. (2008). Antidepressant drugs 'work', but they are not clinically effective. Br. J. Hosp. Med. (Lond.) $69,359$. 
Kirsch, I., Deacon, B. J., Huedo-Medina, T. B., Scoboria, A., Moore, T. J., and Johnson, B. T. (2008). Initial severity and antidepressant benefits: a metaanalysis of data submitted to the Food and Drug Administration. PLoS Med. 5:e45. doi: 10.1371/ journal.pmed.0050045

Knubben, K., Reischies, F. M., Adli, M., Schlattmann, P., Bauer, M., and Dimeo, F. (2007). A randomised, controlled study on the effects of a short-term endurance training programme in patients with major depression. Br. J. Sports Med. 41, 29-33.

Krogh, J., Saltin, B., Gluud, C., and Nordentoft, M. (2009). The DEMO trial: a randomized, parallelgroup, observer-blinded clinical trial of strength versus aerobic versus relaxation training for patients with mild to moderate depression. J. Clin. Psychiatry 70, 790-800.

Krogh, J., Videbech, P., Thomsen, C., Gluud, C., and Nordentoft, M. (2012). DEMO-II trial. Aerobic exercise versus stretching exercise in patients with major depression-a randomised clinical trial. PLoS ONE 7:e48316. doi: 10.1371/journal.pone.0048316

Martinsen, E. W. (1994). Physical activity and depression: clinical experience. Acta Psychiatr. Scand. Suppl. 377, 23-27.

National Institute for Health and Clinical Excellence. (2009). Depression: the treatment and management of depression in adults (update). Available at: http:// www.nice.org.uk/guidance/CG90
Parker, G. (2005). Beyond major depression. Psychol. Med. 35, 467-474.

Rethorst, C. D., Wipfli, B. M., and Landers, D. M. (2009). The antidepressive effects of exercise: a meta-analysis of randomized trials. Sports Med. 39, 491-511.

Rimer, J., Dwan, K., Lawlor, D. A., Greig, C. A., McMurdo, M., Morley, W., et al. (2012). Exercise for depression. Cochrane Database Syst. Rev. 7, CD004366.

Salum, G.A., Manfro, G. G., and Fleck, M.P.(2011). What is not "effective" in mild to moderate depression: antidepressants or the Hamilton Rating Scale for depression? CNS Spectr. PMID: 21536003. [Epub ahead of print].

Santor, D. A., and Coyne, J. C. (2001). Examining symptom expression as a function of symptom severity: item performance on the Hamilton Rating Scale for Depression. Psychol. Assess. 13, 127-139.

Schuch, F. B., Vasconcelos-Moreno, M. P., Borowsky, C., and Fleck, M. P. (2011). Exercise and severe depression: preliminary results of an add-on study. J. Affect. Disord. 133, 615-618.

Searle, A., Calnan, M., Lewis, G., Campbell, J., Taylor, A., and Turner, K. (2011). Patients' views of physical activity as treatment for depression: a qualitative study. Br. J. Gen. Pract. 61, 149-156.

Seime, R. J., and Vickers, K. S. (2006). The challenges of treating depression with exercise: from evidence to practice. Clin. Psychol. 13, 194-197.

Trivedi, M. H., Greer, T. L., Church, T. S., Carmody, T. J., Grannemann, B. D., Galper, D. I., et al. (2011).
Exercise as an augmentation treatment for nonremitted major depressive disorder: a randomized, parallel dose comparison. J. Clin. Psychiatry 72, 677-684.

Trivedi, M.H., Greer, T. L., Grannemann, B. D., Chambliss, H. O., and Jordan, A. N. (2006a). Exercise as an augmentation strategy for treatment of major depression. J. Psychiatr. Pract. 12, 205-213.

Trivedi, M. H., Greer, T. L., Grannemann, B. D., Church, T. S., Galper, D. I., Sunderajan, P., et al. (2006b). TREAD: treatment with exercise augmentation for depression: study rationale and design. Clin. Trials 3, 291-305.

Received: 15 March 2013; accepted: 15 March 2013; published online: 02 April 2013.

Citation: Schuch FB and de Almeida Fleck MP (2013) Is exercise an efficacious treatment for depression? A Comment upon recent negative findings. Front. Psychiatry 4:20. doi: 10.3389/fpsyt.2013.00020

This article was submitted to Frontiers in Affective Disorders and Psychosomatic Research, a specialty of Frontiers in Psychiatry.

Copyright (c) 2013 Schuch and de Almeida Fleck. This is an open-access article distributed under the terms of the Creative Commons Attribution License, which permits use, distribution and reproduction in other forums, provided the original authors and source are credited and subject to any copyright notices concerning any third-party graphics etc. 